\title{
Local circuit model of the subthalamo-pallidal network for the generation of parkinsonian oscillations
}

\author{
Osamu Shouno ${ }^{1,2^{*}}$, Kenji Doya ${ }^{1}$ \\ From The Twenty Third Annual Computational Neuroscience Meeting: CNS*2014 \\ Québec City, Canada. 26-31 July 2014
}

Parkinson's disease is psychomotor dysfunctions caused by degeneration of nigral dopaminegic neurons. Increasing evidence suggests that abnormally synchronized oscillatory activity in the basal ganglia contributes to the expression of parkinsonian motor symptoms. Experimental works demonstrated that neurons in the subthalamic nucleus (STN) and the internal (GPi) and external segments of the globus pallidus (GPe) of dopamine-depleted animals exhibit enhanced oscillatory burst discharges in 8-15 Hz, and suggest that interactions between STN and GPe generate these pathological oscillations [1]. However, the biophysical and neuronal circuitry mechanisms for the generation of these abnormal oscillations by the dopamine depletion remain poorly understood. We addressed this problem by computer simulations of spiking neuron models of the local STN-GPe circuitries with the biophysical properties of neurons and synaptic connections [2-4]. These simulations revealed that the connection patterns between STN and GPe neurons, the high maximal conductance of GPe-to-STN synapses, a type of short-term plasticity at the glutamatergic synapses of GPe neurons, and the reduced baseline firing activity of GPe neurons were critical for generation of oscillatory burst discharges in $10-15 \mathrm{~Hz}$. These latter two conditions were consistent with experimental evidence [4-6]. Characterization of the mechanisms underlying these oscillations suggest that a moderate level of STN post-inhibitory rebound excitation shapes the $10-15 \mathrm{~Hz}$ oscillatory bursts, and that strengthened GPe-STN connectivity and reduced autonomous firing of GPe neurons are critical for the induction of the STN rebound

\footnotetext{
* Correspondence: shouno@oist.jp

'Okinawa Institute of Science and Technology Graduate University, Tancha, Onna-son, Okinawa 904-0495, Japan

Full list of author information is available at the end of the article
}

excitation. These results suggest that the physiological and structural changes in the local subthalamo-pallidal circuits caused by the dopamine depletion underlie the induction of the $10-15 \mathrm{~Hz}$ parkinsonian oscillations in the STN.

\section{Authors' details}

'Okinawa Institute of Science and Technology Graduate University, Tancha, Onna-son, Okinawa 904-0495, Japan. ${ }^{2}$ Honda Research Institute Japan Co., Ltd., Honcho, Wako, Saitama 351-0188, Japan.

Published: 21 July 2014

References

1. Tachibana Y, Iwamuro H, Kita H, Takada M, Nambu A: Subthalami-pallidal interactions underlying parkinsonian neuronal oscillations in the primate basal ganglia. Eur J Neurosci 2011, 34:1470-1484

2. Otsuka T, Abe T, Tsukagawa T, Song WJ: Conductance-based model of the voltage-dependent generation of a plateau potential in subthalamic neurons. J Neurophysiol 2004, 92:255-264.

3. Hanson JE, Jaeger D: Short-Term Plasticity Shapes the Response to Simulated Normal and Parkinsonian Input Patterns in the Globus Pallidus. J Neurosci 2002, 22:5164-5172.

4. Atherton JF, Menard A, Urbain N, Bevan MD: Short-term Depression of External Globus Pallidus- Subthalamic Nucleus Synaptic Transmission and Implications for Patterning Subthalamic Activity. J Neurosci 2013, 33:7130-7144.

5. Fan KY, Baufreton J, Surmeier DJ, Chan CS, Bevan MD: Proliferation of External Globus Pallidus-Subthalamic Nucleus Synapses following Degeneration of Midbrain Dopamine Neurons. J Neurosci 2012, 32:13718-13728.

6. Chan CS, Glajch KE, Gertler TS, Guzman JN, Mercer JN, Lewis AS, Goldberg AB, Tkatch T, Shigemoto R, Fleming SM, et al: HCN channelopathy in external globus pallidus neurons in models of Parkinson's disease. Nat Neurosci 2011, 14:85-92.

doi:10.1186/1471-2202-15-S1-P168

Cite this article as: Shouno and Doya: Local circuit model of the subthalamo-pallidal network for the generation of parkinsonian oscillations. BMC Neuroscience 2014 15(Suppl 1):P168.
() Biomed Central

(c) 2014 Shouno and Doya; licensee BioMed Central Ltd. This is an Open Access article distributed under the terms of the Creative Commons Attribution License (http://creativecommons.org/licenses/by/4.0), which permits unrestricted use, distribution, and reproduction in any medium, provided the original work is properly cited. The Creative Commons Public Domain Dedication waiver (http://creativecommons.org/publicdomain/zero/1.0/) applies to the data made available in this article, unless otherwise stated. 